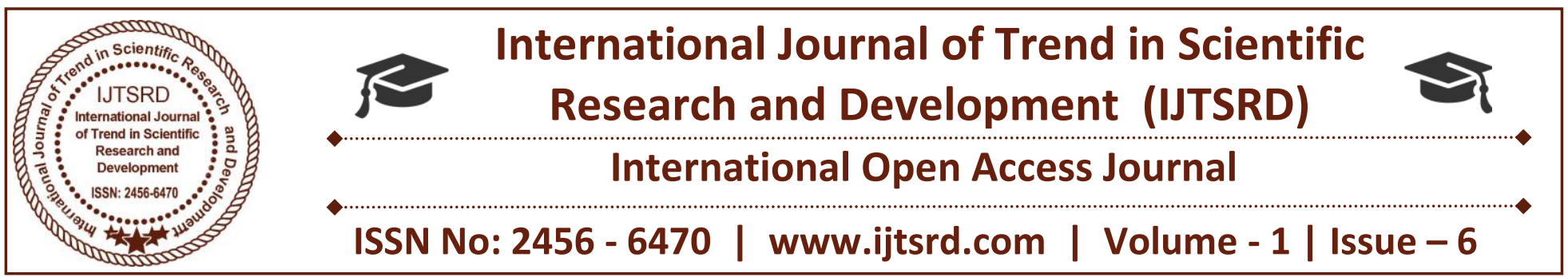

\title{
Design Issues and Applications of Wireless Sensor Network
}

\section{ABSTRACT}

Efficient design and implementation of wireless sensor networks has become a hot area of research in recent years, due to the vast potential of sensor networks to enable applications that connect the physical world to the virtual world. By networking large numbers of tiny sensor nodes, it is possible to obtain data about physical phenomena that was difficult or impossible to obtain in more conventional ways. In future as advances in micro-fabrication technology allow the cost of manufacturing sensor nodes to continue to drop, increasing deployments of wireless sensor networks are expected, with the networks eventually growing to large numbers of nodes. Potential applications for such large-scale wireless sensor networks exist in a variety of fields, including medical monitoring, environmental monitoring, surveillance, home security, military operations, and industrial machine monitoring etc.

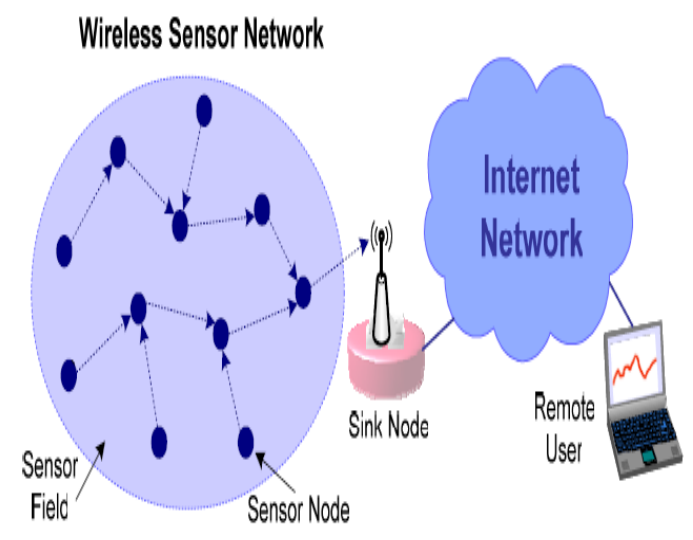

Fig (a) A Typical Sensor Network Architecture

\author{
R. Srilalitha \\ PG Student, Department of Commerce with Computer \\ Applications, Dr.SNS Rajalakshmi College of Arts \\ and Science, Coimbatore, TamilNadu, India
}

\section{INTRODUCTION}

Wireless sensor network (WSN)[5] are highly distributed networks of small, lightweight wireless nodes, deployed in large numbers to monitor the environment or system by the measurement of physical parameters such as temperature, pressure humidity, sound, vibration, pollutants, and collectively relay their sensed data to the sink node. Each node in the networks are connected to each other. Each sensor node in the network consists of three subsystems:

The sensor subsystem is used to sense the environment. The processing subsystem performs the local computations on the sensed data and the communication subsystem is responsible for sharing the sensed data with the neighboring sensor nodes.

The sensor subsystem senses the data in the environment and the processing subsystem processes the sensed data where as the communication subsystem sends the aggregated data to the sink node. A sensor network consists of different types of sensors such as seismic, thermal, visual, and infrared. The size of a sensor node may vary from micro to macro and the cost varies from one to few hundred dollars. The sensor nodes communicate through wireless with in the short distance. The competence of each individual sensor node is limited and the comprehensive power of the entire network is adequate for the required task. The sensor nodes are organized themselves autonomously into a wireless network. Sensor nodes operated in the battery power expected to sense for a long time. In most of the 
situations, the battery cannot be recharged or replaced.

Wireless Sensor Networks (WSN) are intended for monitoring an environment. The main task of a wireless sensor node is to sense and collect data from a certain domain, process them and transmit it to the sink where the application lies.

Sensor networks have emerged as a promising tool for monitoring (and possibly actuating) the physical world, utilizing self-organizing networks of batterypowered wireless sensors that can sense, process and communicate. In sensor networks, energy is a critical resource, while applications exhibit a limited set of characteristics. Thus, there is both a need and an opportunity to optimize the network architecture for the applications in order to minimize resource consumed. The requirements and limitations of sensor networks make their architecture and protocols both challenging and divergent from the needs of traditional internet architecture.

A sensor network [1][3] is a network of many tiny disposable low power devices, called nodes, which are spatially distributed in order to perform an application-oriented global task. These nodes form a network by communicating with each other either directly or through other nodes. One or more nodes among them will serve as sink(s) that are capable of communicating with the user either directly or through the existing wired networks. The primary component of the network is the sensor, essential for monitoring real world physical conditions such as sound, temperature, humidity, intensity, vibration, pressure, motion, pollutants etc. at different locations.

\section{IMPORTANT REQUIREMENTS OF A WSN}

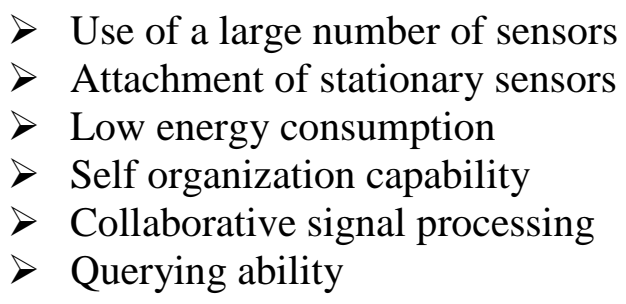

\section{DESIGN ISSUES IN WIRELESS SENSOR NETWORK}

Despite the innumerable applications of WSNs, these networks have several restrictions, such as limited energy supply, limited computing power, and limited bandwidth of the wireless links connecting sensor nodes. One of the main goals of WSNs is to carry out data communication while trying to prolong the lifetime of the network and prevent connectivity degradation by employing aggressive energy management techniques. The design of routing protocols in WSNs [5][6] is influenced by many challenging factors. These factors must be overcome before efficient communication can be achieved in WSNs. In the following, we summarize some of the routing challenges and design issues that affect the routing process in WSNs.

\section{Node deployment}

Node deployment [4] in WSNs is applicationdependent and can be either manual (deterministic) or randomized. In manual deployment, the sensors are manually placed and data is routed through predetermined paths. However, in random node deployment, the sensor nodes are scattered randomly, creating an ad hoc routing infrastructure. If the resultant distribution of nodes is not uniform, optimal clustering becomes necessary to allow connectivity and enable energy-efficient network operation. Inter sensor communication is normally within short transmission ranges due to energy and bandwidth limitations. Therefore, it is most likely that a route will consist of multiple wireless hops.

\section{Energy consumption without losing accuracy}

Sensor nodes can use up their limited supply of energy performing computations and transmitting information in a wireless environment. As such, energy-conserving forms of communication and computation are essential. Sensor node lifetime shows a strong dependence on battery lifetime. In a multihop WSN, each node plays a dual role as data sender and data router. The malfunctioning of some sensor nodes due to power failure can cause significant topological changes, and might require rerouting of packets and reorganization of the network.

\section{Data reporting method}

Data reporting [2] in WSNs is application-dependent and also depends on the time criticality of the data. Data reporting can be categorized as either timedriven, event driven, query-driven, or a hybrid of all these methods. The time-driven delivery method is suitable for applications that require periodic data monitoring. As such, sensor nodes will periodically switch on their sensors and transmitters, sense the environment, and transmit the data of interest at constant periodic time intervals. In event-driven and 
query-driven methods, sensor nodes react immediately to sudden and drastic changes in the value of a sensed attribute due to the occurrence of a certain event, or respond to a query generated by the $\mathrm{BS}$ or another node in the network. As such, these are well suited to time-critical applications. A combination of the previous methods is also possible. The routing protocol is highly influenced by the data reporting method in terms of energy consumption and route calculations.

\section{Node/link heterogeneity}

In many studies, all sensor nodes were assumed to be homogeneous (i.e., have equal capacity in terms of computation, communication, and power). However, depending on the application a sensor node can have a different role or capability. The existence of a heterogeneous set of sensors raises many technical issues related to data routing. For example, some applications might require a diverse mixture of sensors for monitoring temperature, pressure, and humidity of the surrounding environment, detecting motion via acoustic signatures, and capturing images or video tracking of moving objects. Either these special sensors can be deployed independently or the different functionalities can be included in the same sensor nodes. Even data reading and reporting can be generated from these sensors at different rates, subject to diverse QoS constraints, and can follow multiple data reporting models. For example, hierarchical protocols designate a cluster head node different from the normal sensors. These cluster heads can be chosen from the deployed sensors or be more powerful than other sensor nodes in terms of energy, bandwidth, and memory. Hence, the burden of transmission to the Base Station (BS) is handled by the set of cluster heads.

\section{Fault tolerance}

Some sensor nodes [11]may fail or be blocked due to lack of power, physical damage, or environmental interference. The failure of sensor nodes should not affect the overall task of the sensor network. If many nodes fail, medium access control (MAC) and routing protocols must accommodate formation of new links and routes to the data collection BSs. This may require actively adjusting transmit powers and signaling rates on the existing links to reduce energy consumption, or rerouting packets through regions of the network where more energy is available.
Therefore, multiple levels of redundancy may be needed in a fault-tolerant sensor network.

\section{Scalability}

The number of sensor nodes deployed in the sensing area may be on the order of hundreds or thousands, or more. Any routing scheme must be able to work with this huge number of sensor nodes. In addition, sensor network routing protocols[12] should be scalable enough to respond to events in the environment. Until an event occurs, most sensors can remain in the sleep state, with data from the few remaining sensors providing coarse quality.

\section{Network dynamics}

In many studies, sensor nodes are assumed to be fixed. However, in many applications both the BS or sensor nodes can be mobile. As such, routing messages from or to moving nodes is more challenging since route and topology stability become important issues, in addition to energy, bandwidth, and so forth. Moreover, the phenomenon can be mobile (e.g., a target detection/ tracking application). On the other hand, sensing fixed events allows the network to work in a reactive mode (i.e., generating traffic when reporting), while dynamic events in most applications require periodic reporting to the BS.

\section{Transmission media}

In a multihop sensor network [30], communicating nodes are linked by a wireless medium. The traditional problems associated with a wireless channel (e.g., fading, high error rate) may also affect the operation of the sensor network. In general, the required bandwidth of sensor data will be low, on the order of $1-100 \mathrm{~kb} / \mathrm{s}$. Related to the transmission media is the design of MAC. One approach to MAC design for sensor networks is to use time-division multiple access (TDMA)-based protocols that conserve more energy than contention-based protocols like carrier sense multiple access (CSMA) (e.g., IEEE 802.11) [10]. Bluetooth technology can also be used.

\section{Connectivity}

High node density in sensor networks precludes them from being completely isolated from each other. Therefore, sensor nodes are expected to be highly connected. This, however, may not prevent the network topology from being variable and the 
network size from shrinking due to sensor node failures. In addition, connectivity depends on the possibly random distribution of nodes.

\section{Coverage}

In WSNs, each sensor node obtains a certain view of the environment. A given sensor's view of the environment is limited in both range and accuracy and it can only cover a limited physical area of the environment. Hence, area coverage is also an important design parameter in WSNs.

\section{Data aggregation}

Since sensor nodes may generate significant redundant data, similar packets from multiple nodes can be aggregated[6] to reduce the number of transmissions. Data aggregation is the combination of data from different sources according to a certain aggregation function (e.g., duplicate suppression, minima, maxima, and average). This technique has been used to achieve energy efficiency and data transfer optimization in a number of routing protocols. Signal processing methods can also be used for data aggregation. In this case, it is referred to as data fusion[6][10] where a node is capable of producing a more accurate output signal by using some techniques such as beam forming to combine the incoming signals and reducing the noise in these signals.

\section{Quality of service}

In some applications, data should be delivered within a certain period of time from the moment it is sensed, or it will be useless. Therefore, bounded latency for data delivery is another condition for time-constrained applications. However, in many applications, conservation of energy, which is directly related to network lifetime, is considered relatively more important than the quality of data sent. As energy is depleted, the network may be required to reduce the quality of results in order to reduce energy dissipation in the nodes and hence lengthen the total network lifetime. Hence, energy-aware routing protocols are required to capture this requirement.

\section{FEATURES OF WIRELESS NETWORK \\ SENSOR}

Protocol design for sensor networks must account for the properties of ad hoc networks, including the following:
$>$ Lifetime constraints imposed by the limited energy supplies of the nodes in the network.

$>$ Unreliable communication due to the wireless medium.

Need for self-configuration, requiring little or no human intervention.

\section{PROTOCOLS USED IN WSN}

Routing protocols [1] can be classified into

$>$ Multipath-based protocol

$>$ Query-based protocol

$>$ Negotiation- based protocol

$>$ QoS-based, or coherent-based protocol

\section{TABLE I: ROUTING PROTOCOLS FOR WSNS}

\begin{tabular}{|c|c|}
\hline Category & Representative Protocols \\
\hline $\begin{array}{l}\text { Location-based } \\
\text { Protocols }\end{array}$ & $\begin{array}{l}\text { MECN, SMECN, GAF, GEAR, } \\
\text { Span, TBF, BVGF, GeRaF }\end{array}$ \\
\hline $\begin{array}{l}\text { Data-centric } \\
\text { Protocols }\end{array}$ & $\begin{array}{l}\text { SPIN, Directed Diffusion, Rumor } \\
\text { Routing, COUGAR,ACQUIRE, } \\
\text { EAD, Information-Directed } \\
\text { Routing(DD), Gradient-Based } \\
\text { Routing(GBR), Energy-aware } \\
\text { Routing, Information-Directed } \\
\text { Routing, } \\
\text { Information Dissemination, Home } \\
\text { Agent Based Information } \\
\text { Dissemination }\end{array}$ \\
\hline $\begin{array}{l}\text { Hierarchical } \\
\text { Protocols }\end{array}$ & $\begin{array}{l}\text { LEACH, PEGASIS, HEED, TEEN, } \\
\text { APTEEN }\end{array}$ \\
\hline $\begin{array}{l}\text { Mobility-based } \\
\text { Protocols }\end{array}$ & $\begin{array}{l}\text { SEAD, TTDD, Joint Mobility and } \\
\text { Routing, Data MULES Dynamic } \\
\text { Proxy Tree-Base } \\
\text { Dissemination }\end{array}$ \\
\hline $\begin{array}{l}\text { Heterogeneity- } \\
\text { based Protocols }\end{array}$ & IDSQ, CADR, CHR \\
\hline $\begin{array}{l}\text { Multipath- } \\
\text { based Protocols }\end{array}$ & $\begin{array}{l}\text { Sensor-Disjoint Multipath, Braided } \\
\text { Multipath, N-to-1 } \\
\text { Multipath Discovery }\end{array}$ \\
\hline $\begin{array}{l}\text { QoS-based } \\
\text { protocols }\end{array}$ & $\begin{array}{lll}\text { SAR, } & \text { SPEED, } & \text { Energy-aware } \\
\text { routing } & & \end{array}$ \\
\hline
\end{tabular}




\section{APPLICATIONS OF WIRELESS SENSOR NETWORK}

Sensor networks may consist of many different types of sensors[4] such as seismic, low sampling rate magnetic, visual, thermal, infra-red, acoustic and radar, which are able to monitor a wide variety of ambient conditions. Sensor nodes can be used for continuous sensing, event detection, event ID, and local control of actuators. The concept of micro sensing and wireless connection of these nodes promise many new application areas. The applications are categorized into military, environmental, health, home, and other commercial areas.

\section{Military Applications}

Wireless sensor networks can be an integral part of military command,[2] control, communication, computing, intelligence, surveillance and targeting (C4ISRT) systems. The rapid deployment, fault tolerance and self organization characteristics of sensor networks make them a very promising sensing technique for military (C4ISRT). Since sensor networks are based on dense deployment of disposable and low cost sensor nodes, destruction of some nodes by hostile actions does not affect military applications as much as the destruction of traditional sensor, which makes sensor networks concept a better approach for battlefield. Various military applications of sensor networks are monitoring friendly forces, equipments and ammunition, biological and chemical attack detection and reconnaissance.

\section{Environmental Applications}

Some environmental applications[14] of sensor network include tracking the movement of birds, small animals and insects, monitoring environmental conditions that affect crops and livestock ,irrigation; macro instruments for large scale earth monitoring and planetary exploration, chemical/biological detection, precision agriculture, biological, earth and environmental monitoring in marine, soil and atmosphere, forest fire detection meteorological and geo physical research, flood detection, bio complexity mapping of the environment and pollution study.

\section{Health Application}

Some of the applications are providing interfaces for the disabled, integrated patient monitoring, diagnostics, drug administration in hospital, monitoring the movements and internal process of insects[33] or other small animals, telemonitoring of human physiological data, and tracking and monitoring doctors and patients inside a hospital.

\section{Home Applications}

A technology advances, in home automation smart sensor nodes and actuators can be buried appliances such as vacuum cleaners, microwave ovens, refrigerators and VCRs. These sensor nodes inside the domestic devices can interact with each other and with a external network via the internet or satellite. They allow end users to manage home devices locally and remotely more easily.

\section{Other Commercial applications}

Some of the commercial applications[4] are monitoring material fatigue, building virtual keyboards, managing inventory, monitoring product quality, constructing smart office spaces, environmental control in office buildings, robot control and guidance in automatic manufacturing environment, interactive toys and museums, factory process control and automation, monitoring disaster area, smart structures with sensor nodes embedded inside, machine diagnosis, transportation, factory instrumentation, local control of actuators, detecting and monitoring car thefts, vehicle detection and tracking, instrumentation of semiconductor processing chambers, rotating machinery, wind tunnels and anechoic chambers.

\section{CONCLUSION}

Wireless Sensor nodes are densely deployed either very close or directly inside the phenomenon to be observed. Therefore, they usually work unattended in remote geographic areas. . In most of the sensor network applications replenishment of power resources is nearly impossible. Another important factor that influences the consumption of more power in sensor networks is that each sensor node consumes power not only for sensing but also for processing the sensed data and transmitting/receiving them to/from neighbors. WSN is the one of the most challenging task for researchers nowadays. Some important factors should be taken into account when designing an energy efficient routing protocol for WSN. 


\section{REFERENCES}

1) Akkaya, K., and Younis, M. (2005), “A Survey on Routing Protocols for Wireless Sensor Networks", Elsevier Journel of Ad Hoc Networks, Vol. 3, pp. 325-349.

2) Akyildiz, I.F., Vuran, M.C., Akan, O., and Su, W. (2004), "Wireless Sensor Networks:A Survey Revisited", Elsevier Journel of Computer Networks, Vol. 45, No. 3.

3) Akyildiz, I. F., Su, W., Sankarasubramaniam, Y., and Cayirci, E., (2002), "Wireless sensor networks: a survey", Elsevier Journal of Computer Networks, Vol. 38, No. 4, pp. 393-422.

4) Al-Karaki, J.N., Kamal, A.E., (2004), "Routing Techniques in Wireless Sensor Networks: A Survey", IEEE transactions on Wireless Communications, Vol.11, No. 6, pp. 6-28.

5) Akyildiz, I. F., Su, W., Sankarasubramaniam, Y., and Cayirci, E., (2002), "A survey on sensor networks", IEEE Communications Magazine, Vol. 40, No. 8, pp.102-114.

6) Azni, A.H., Madihah Mohd, S., Azreen, A., and Ariff Syah, J., (2009), "Performance Analysis of Routing Protocol for WSN Using Data Centric Approach", World Academy of Science, Engineering and Technology, Vol. 53. 